\title{
Phonon Dispersion for Armchair and Zigzag Carbon Nanotubes
}

\author{
Rasmita Sahoo, Rashmi Ranjan Mishra \\ Department of Physics, Birla Institute of Technology and Science, Pilani, Rajasthan, India \\ Email: sahoorasmita@yahoo.com
}

Received 17 February 2014; revised 18 March 2014; accepted 2 April 2014

Copyright (C) 2014 by authors and Scientific Research Publishing Inc.

This work is licensed under the Creative Commons Attribution International License (CC BY). http://creativecommons.org/licenses/by/4.0/

c) (i) Open Access

\begin{abstract}
In this paper we present calculations of phonon dispersion of different types of single walled carbon nanotubes (SWCNT). In these calculations, the starting point has been the phonon dispersion of graphene. Zone folding scheme is then applied to obtain the phonon dispersion of CNT from that of graphene. Since the radial breathing modes in the phonon dispersion are not obtained by the zone-folding method, certain modifications are made in the lower branches to obtain these modes. These modifications produce results similar to those obtained by more sophisticated ab-initio methods.
\end{abstract}

\section{Keywords}

\section{Carbon Nanotubes, Zone Folding, Phonon Dispersion}

\section{Introduction}

After the discovery of carbon nanotubes in 1991 [1], much attention has been given to the investigation of their electrical, vibrational and thermal properties [2]-[6]. Phonon dispersion plays a fundamental role in determining both thermal and electrical properties of carbon nanotubes. In this paper we present some of our calculations of phonon dispersion of armchair and zigzag CNTs. As CNTs are rolled up by graphene, many physical properties of carbon nanotubes are closely related to those of graphene and to some extent, graphite. In our calculation of phonon dispersion of CNT, the starting point is that for grapheme, from which, the phonon dispersions of different CNTs are obtained through the zone folding scheme. Since the radial breathing modes in the phonon dispersions are not obtained by the zone-folding method, certain modifications are made in the lower branches to obtain these modes. The results obtained after these modifications are very similar to those produced through more sophisticated methods. The paper is organized as follows. In section 2 a brief description of the structure of CNT is given. The zone folding method, which is used to calculate the phonon dispersion of CNT from that 
of graphene, is discussed in section 3 and the results of calculations are presented in Section 4. Section 5 concludes the paper.

\section{Structure of CNT}

The lattice parameters of CNT in terms of the lattice structure of graphene, from which it can be imagined to be obtained by rolling, are shown in Figure 1 below.

In the figure, OADB is a strip of graphene that is folded to form a CNT. Equivalent sites on the opposite edges, such as sites $\mathrm{O}$ and $\mathrm{A}$, and sites $\mathrm{B}$ and $\mathrm{D}$, are joined as a result of folding. The minimum translation along the tube, so that the structure is repeated, is OT. So, the primitive lattice translation vectors of CNT are the chiral vector $\vec{C}_{h}$, and the translation vector $\vec{T}$, which in terms of graphene primitive vectors, are given as:

and

$$
\vec{C}_{h}=n \vec{a}_{1}+m \vec{a}_{2}
$$

$$
\vec{T}=t_{1} \vec{a}_{1}+t_{2} \vec{a}_{2} .
$$

Here $n, m, t_{1}$ and $t_{2}$ are integers. The CNT that is obtained by folding the strip OADB will thus consist of two unit cells. In the reciprocal space, the two primitive translation vectors, $\vec{K}_{1}$ and $\vec{K}_{2}$ as shown in Figure 2, are given as:

$$
\begin{aligned}
& \vec{K}_{1}=\frac{\left(-t_{2} \vec{b}_{1}+t_{1} \vec{b}_{2}\right)}{N}, \\
& \vec{K}_{2}=\frac{\left(m \vec{b}_{1}-n \vec{b}_{2}\right)}{N}
\end{aligned}
$$

where $N$ is the number of hexagons per unit cell of a CNT and $\vec{b}_{1}, \vec{b}_{2}$ are the reciprocal primitive lattice

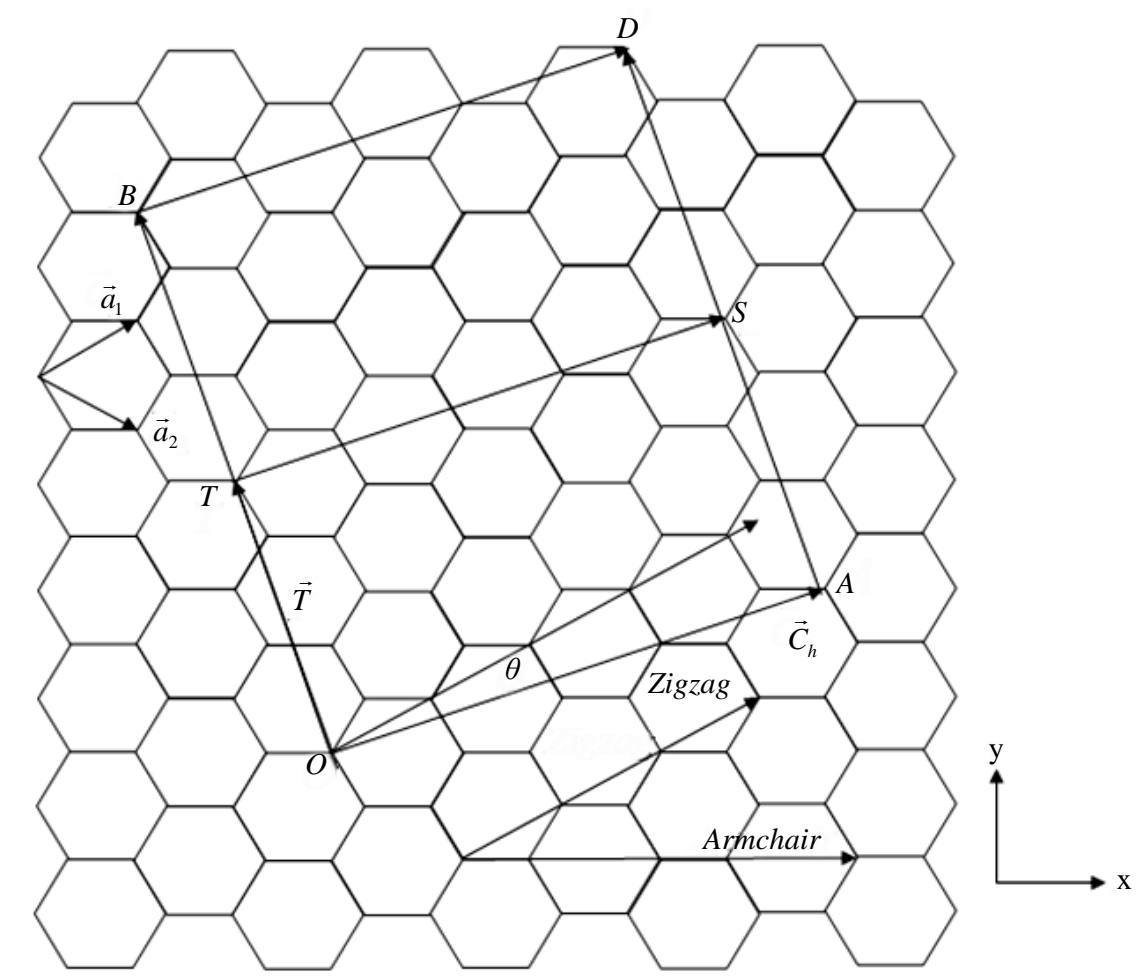

Figure 1. The unrolled honeycomb lattice of a nanotube showing the chiral vector $\vec{C}_{h}$ and the translational vector $\vec{T}$ of a $(4,1)$ nanotube. 


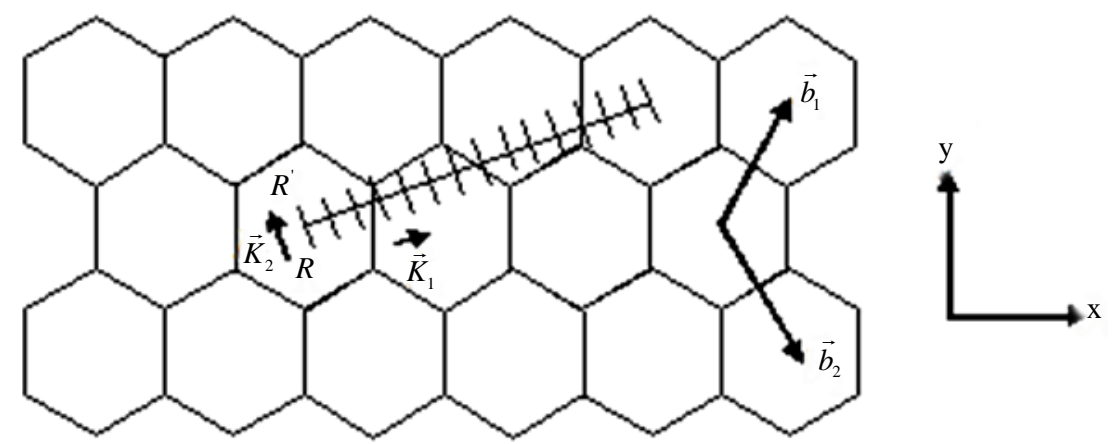

Figure 2. Extended reciprocal zone for $(4,1)$ CNT superimposed on the graphene reciprocal lattice.

vectors of graphene.

As $N \vec{K}_{1}$ is a reciprocal lattice vector of the $2 D$ graphene and $t_{1}$ and $t_{2}$ do not have a common divisor except unity, none of the $\mathrm{N}$ vectors, $q \vec{K}_{1}(q=0, \cdots, N-1)$ is a reciprocal lattice vector of the $2 D$ graphene. Thus the one dimensional Brillouin zone of CNT is a line segment $\left(R R^{\prime}\right)$ of length $2 \pi / T$ along the $\vec{K}_{2}$ vector in the reciprocal space of graphene. However, there are $N$ copies of it, along with $\vec{K}_{1}$, repeated at $q \vec{K}_{1}$. The totality of all these line segments in the $k$-space of graphene, forms the Brillouin zone of CNT. The $\vec{k}$ vectors form a continuum along with $\vec{K}_{2}$, and are discrete along $\vec{K}_{1}$. This structure of the k-space of CNTs forms the basis for the zone-folding scheme discussed in the next section.

\section{The Zone Folding Scheme}

As discussed in the previous section, the Brillouin zone of CNT consists of a number of line segments in the reciprocal space of graphene, oriented along one of the reciprocal lattice vector (let $\vec{K}_{2}$ ) of CNT. Further the number of line segments is equal to the number of hexagons in a unit cell of CNT. The existence of several identical line segments is a consequence of the fact that CNT has another folded dimension, the one around the tube. The $k$-vectors of CNT, thus, form a continuum along $\vec{K}_{2}$, as this is the reciprocal lattice vector corresponding to the long dimension, whereas they are discrete along the perpendicular $\vec{K}_{1}$ direction, corresponding to the much shorter, nanoscale dimension, around the tube. A k-vector of CNT can, therefore, be expressed as:

where

$$
k \frac{\vec{K}_{2}}{\left|\vec{K}_{2}\right|}+q \vec{K}_{1},
$$

and $q=0, \cdots, N-1$.

All these k-vectors are distinct k-vectors of graphene. However, $q=N$ is equivalent to $q=0, q=N+1$ is equivalent to $q=1$, and so on, as $N \vec{K}_{1}$ is a reciprocal lattice vector of graphene. Thus, in a simple scheme, the energy dispersion of CNT is taken to be those sections of the energy dispersion of graphene, that are confined to the discrete line segments, comprising the Brillouin zone of CNT. Each of these N lines in the k-space of graphene gives rise to a dispersion curve, or a sub band for CNT. This method, by which the phonon dispersion of CNT is obtained from that of graphene, is known by the name zone folding [4]. The advantage of zone folding method is that it is very fast and can calculate the properties of any desired CNT very quickly in comparison to that done through first principle calculations, and as we show in the present calculation, it brings out most of the features of the dispersion curves as obtained through these more advanced calculations.

\section{Phonon Dispersion of Carbon Nanotube (CNT)}

For calculating phonon dispersion of CNT we have used the zone-folding scheme as described in section 3 . According to this method the phonon dispersion of CNT is given as: 


$$
\omega_{1 D}^{m q}(k)=\omega_{2 D}^{m}\left(k \frac{\vec{K}_{2}}{\left|\vec{K}_{2}\right|}+q \vec{K}_{1}\right)
$$

for $m=1, \cdots, 6 ; q=0, \cdots, N-1$ and

$$
-\frac{\pi}{T} \leq k \leq \frac{\pi}{T}
$$

Here $\omega_{2 D}^{m}$ represents the phonon dispersion relation of $2 D$ graphene, $k$ is the one dimensional wave vector for the one-dimensional CNT, $\vec{K}_{1}$ and $\vec{K}_{2}$ are reciprocal lattice vectors along the circumference and along the axis of the tube respectively, $T$ is the magnitude of the translation vector $\vec{T}$ and $N$ is the number of hexagons per unit cell of a CNT and each of the $N$ number of $q$ values above corresponds to each of the $N$ line segment. As there are $2 N$ carbon atoms in the unit cell of a carbon Nanotube, we have $6 N$ phonon branches in the phonon dispersion diagram of CNT, i.e., each of the six phonon dispersion relation of graphene splits into $N$ branches.

For armchair CNTs, $k_{x}$ is in the circumferential direction and for zigzag CNTs, $k_{y}$ is in circumferential direction. So, using quantization for the wave vectors in these respective directions and using Equation (2), the phonon dispersion relations are obtained for $(10,10)$ armchair CNT and $(10,0)$ zigzag CNT from that of graphene. For calculating the phonon dispersion of graphene, upto 4th nearest neighbour interactions are included as the quadratic nature of the lowest phonon branch comes only after that [7]. The results obtained for armchair as well as zigzag CNTs are shown in Figure 3(a) and Figure 3(b) respectively. As $N=2 n$ for both ( $n, n)$ armchair and $(n, 0)$ zigzag CNTs, there are $6 N$, or equivalently $12 n$, branches present in the phonon dispersion of both types of CNTs.

The results are compared with some previously reported calculations [5], in Figure 4, which shows a good qualitative agreement. In the figure, the first and the fourth represent the present calculations, and the 2nd and 3rd are the earlier reported calculations using the ab-initio method. From the above comparisons it is clear that though we get good agreement with results reported earlier, we do not get the radial breathing mode which is one of the important phonon modes of CNT. This is a drawback of the zone folding method, which does not take into account the effect of curvature in CNT, and radial breathing mode is one such effect. In order to obtain this mode we have made certain ad-hoc modifications to the lower lying acoustic modes. This modification involves shifting up the bottom-most acoustic phonon branch near the $\Gamma$ point, so as to match the frequency at $k=0$, as reported in the literature [6], while, at the same time, ensuring that there is negligible shift at the zone boundary. After this modification we could obtain the RBM branch of phonon dispersion, as well as their k-dependence, as reported in recently published result [8]. According to the literature there should be four acoustic modes present

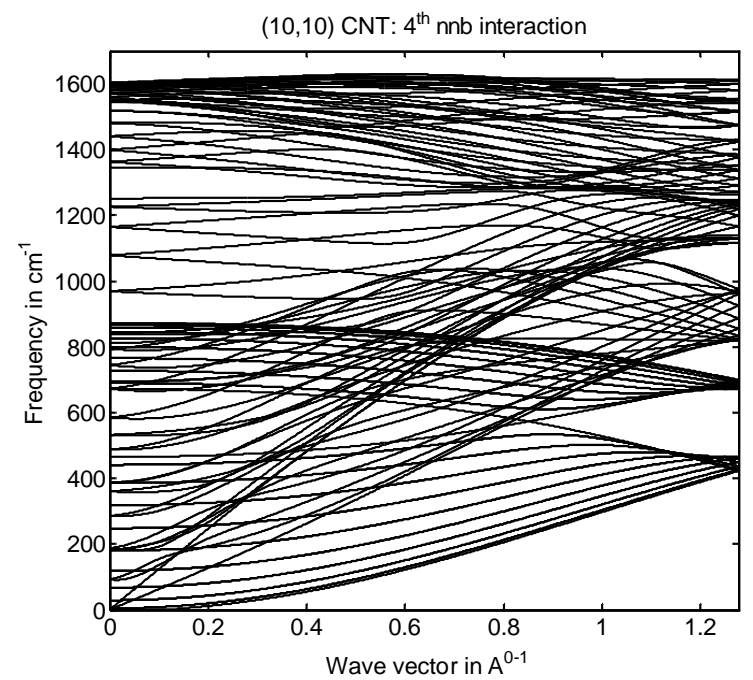

(a)

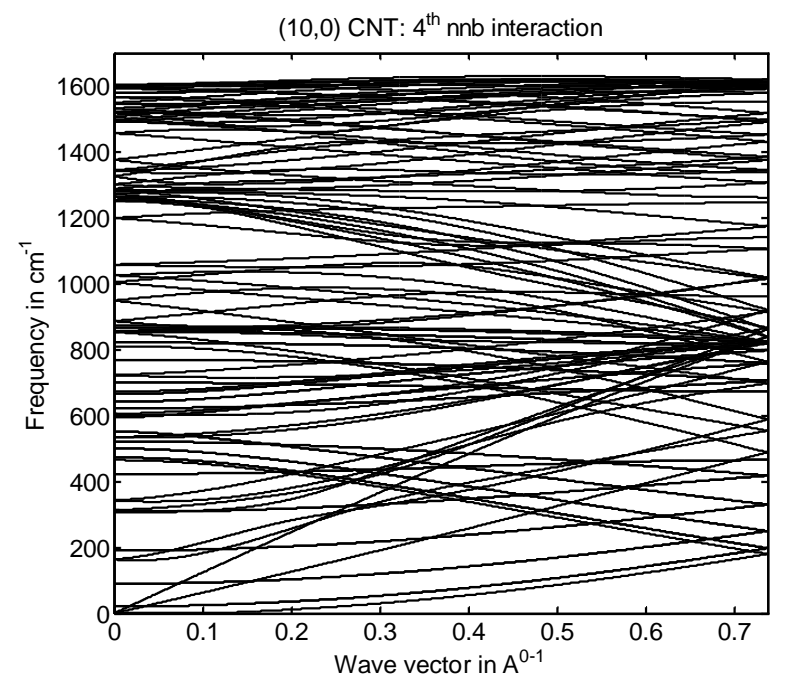

(b)

Figure 3. Phonon dispersion of (a) $(10,10)$ armchair CNT and (b) $(10,0)$ zigzag CNT. 
in CNT phonon dispersion, out of which two are linear in $k$, and two are degenerate and quadratic in $k$. This quadratic dependence at small $k$, is present in our calculation, which appears after including the 4th nearest neighbor interaction in graphene, and then applying the zone folding method, together with the shift mentioned above. Moreover, as reported in the literature [9] [10], there is very little error in the optical branches if one uses zone folding method to obtain the same. As such we are able to obtain all the branches for CNT by using zone folding method with the stated modifications. The results of the modified calculation for $(10,10)$ and $(10,0)$ CNTs are shown in Figure 5. In both the figures the thick lines correspond to the RBM branch and the frequency for these branches varies inversely with the diameter of the corresponding CNT. Further it is clear from the figures that for CNTs there are four acoustic branches out of which two vary linearly with the wave vector and the third one, which is doubly degenerate, exhibits nearly a quadratic dependency on the wave vector. Thus by using zone folding method with the up-shifting, we are able to obtain all the branches of CNT phonon dispersion.

\section{Conclusion}

Phonon dispersions are obtained for both armchair and zigzag CNTs using the simple zone-folding method. The results obtained show good matching with published results that use more sophisticated ab-initio calculations. Thus, we conclude that, for calculation of the qualitative features of phonon dispersion of CNTs, the simple

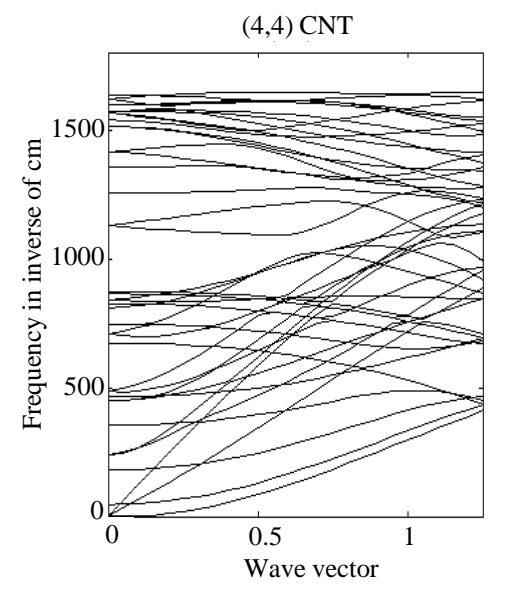

(a)

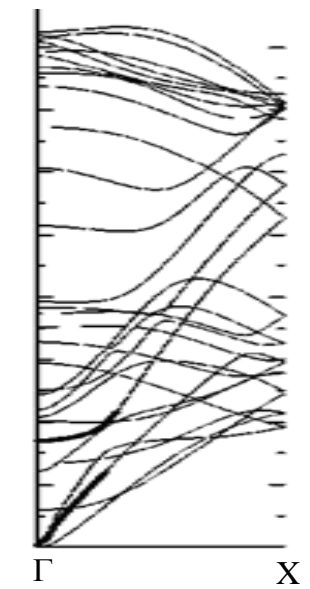

(b)

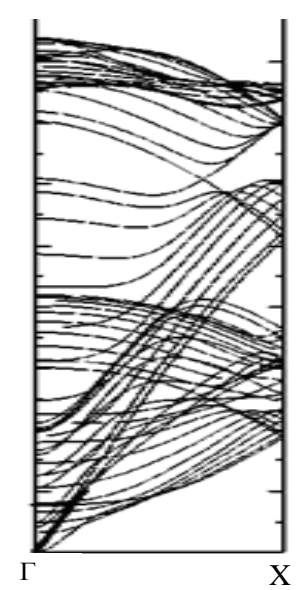

(c)

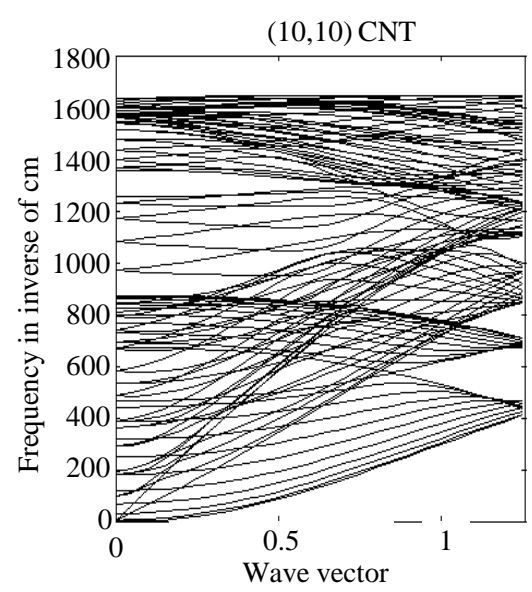

(d)

Figure 4. Phonon dispersions obtained from (a) present calculation for $(4,4)$

CNTs, (b) ab-initio method [5] for $(4,4)$ CNT, (c) ab-initio method [5] for $(10,10)$ CNT and (d) present calculation for $(10,10)$ CNTs. 


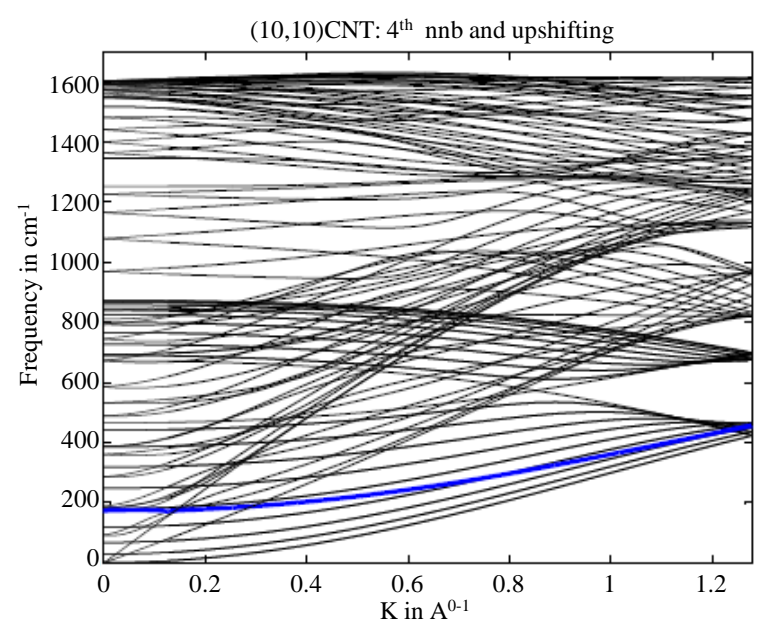

(a)

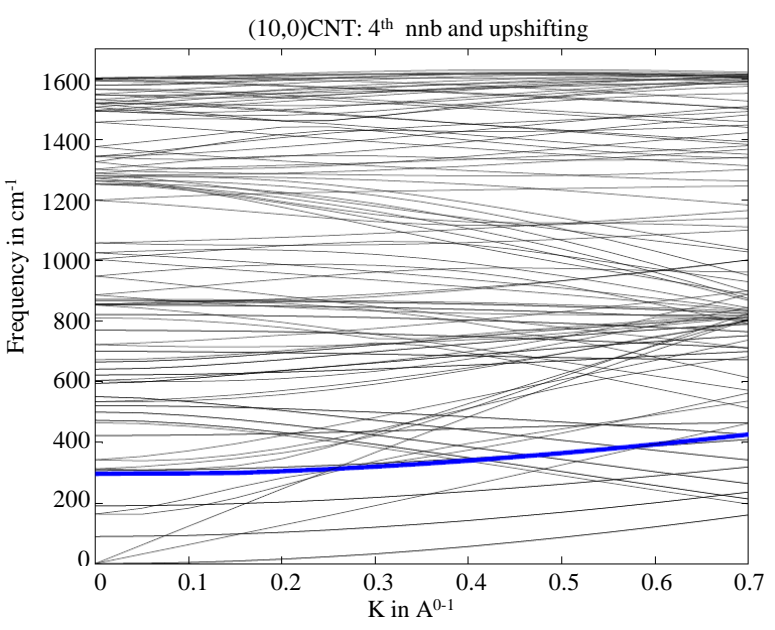

(b)

Figure 5. Phonon dispersion using shifting for (a) $(10,10)$ and (b) $(10,0)$ CNTs.

zone-folding method, which is quicker than these other calculations, is good enough.

\section{Acknowledgements}

The authors would like to thank Prof. Eduardo Barros, Federal University of Ceara, Brazil, for his valuable suggestions.

\section{References}

[1] Iijima, S. (1991) Helical Microtubules of Graphitic Carbon. Nature, 354, 56-58. http://dx.doi.org/10.1038/354056a0

[2] Dubay, O. and Kresse, G. (2003) Accurate Density Functional Calculations for the Phonon Dispersion Relations of Graphite Layer and Carbon Nanotubes. Physical Review B, 67, 035401. http://dx.doi.org/10.1103/PhysRevB.67.035401

[3] Maultzsch, J., Reich, S., Thomsen, C., Dobardzic, E., Milosevic, I. and Damnjanovic, M. (2002) Phonon Dispersion of Carbon Nanotubes. Solid State Communication, 121, 471-474. http://dx.doi.org/10.1016/S0038-1098(02)00025-X

[4] Saito, R., Dresselhaus, M.S. and Dresselhaus, G. (1998) Physical Properties of Carbon Nanotubes. Imperial College Press, London.

[5] Portal, D.S., Artacho, E., Soler, J.M., Rubio, A. and Ordejon, P. (1999) Ab Initio Structural, Elastic, and Vibrational Properties of Carbon Nanotubes. Physical Review B, 59, 12678-12688.

http://dx.doi.org/10.1103/PhysRevB.59.12678

[6] Kurti, J., Kresse, G. and Kuzmany, H. (1998) First-Principles Calculations of the Radial Breathing Mode of Single-Wall Carbon Nanotubes. Physical Review B, 58, R8869-R8872. http://dx.doi.org/10.1103/PhysRevB.58.R8869

[7] Sahoo, R. and Mishra, R.R. (2012) Phonon Dispersion of Graphene Revisited. Journal of Experimental and Theoretical Physics, 114, 805-809. http://dx.doi.org/10.1134/S1063776112040152

[8] Zimmermann, J., Pavone, P. and Cuniberti, G. (2008) Vibrational Modes and Low-Temperature Thermal Properties of Graphene and Carbon Nanotubes: Minimal Force-Constant Model. Physical Review B, 78, 045410.

[9] Lazzeri, M., Piscanec, S., Mauri, F., Ferrari, A.C. and Robertson, J. (2005) Electron Transport and Hot Phonons in Carbon Nanotubes. Physical Review Letters, 95, 236802. http://dx.doi.org/10.1103/PhysRevLett.95.236802

[10] Kauser, M.Z. and Ruden, P.P. (2007) Effects of Chirality and Diameter on the Transport Properties of Semiconducting Carbon Nanotubes. Journal of Applied Physics, 102, 033712. http://dx.doi.org/10.1063/1.2767224 